Cuestiones de Sociología, no 18, e047, junio 2018, ISSN 2346-8904

Universidad Nacional de La Plata

Facultad de Humanidades y Ciencias de la Educación

Departamento de Sociología

\title{
Deporte: la dinámica de lo analizable
}

Garriga Zucal, José

Instituto de Altos Estudios Sociales (IDAES) - Universidad Nacional de San Martín, CONICET, Argentina garrigajose@ hotmail.com

\section{Hang, Julia}

Centro de Investigaciones Sociohistóricas (CISH) -Facultad de Humanidades y Ciencias de la Educación-Universidad Nacional de La Plata - CONICET, Argentina

julita.hang@gmail.com

\section{Iuliano, Rodolfo}

Centro Interdisciplinario de Metodología de las Ciencias Sociales (CIMeCS) - Facultad de Humanidades y Ciencias de la Educación -Universidad Nacional de La Plata, Argentina rodolfoiuliano@gmail.com

Cita sugerida: Garriga Zucal, J., Hang, J. y luliano, R. (2018). Deporte: la dinámica de lo analizable. Cuestiones de Sociología, 18, e047. https://doi.org/10.24215/23468904e047 


\title{
Deporte: la dinámica de lo analizable
}

\author{
José Garriga Zucal \\ Instituto de Altos Estudios Sociales (IDAES) - Universidad \\ Nacional de San Martin, CONICET, Argentina \\ garrigajose@hotmail.com \\ Julia Hang \\ Centro de Investigaciones Sociohistóricas (CISH) - \\ Facultad de Humanidades y Ciencias de la Educación \\ - Universidad Nacional de La Plata - CONICET, \\ Argentina \\ julita.hang@gmail.com \\ Rodolfo Iuliano \\ Centro Interdisciplinario de Metodología de las Ciencias \\ Sociales (CIMeCS) - Facultad de Humanidades y Ciencias \\ de la Educación-Universidad Nacional de La Plata, \\ Argentina \\ rodolfoiuliano@gmail.com
}

El fenómeno deportivo ha sido conceptualizado, desde el campo de las ciencias sociales, a partir de diferentes claves teóricas. Presentaremos aquí cuatro tradiciones gravitantes, cuyas conceptualizaciones recorren el presente dosier. En primer lugar, es posible ubicar a las perspectivas que son tributarias de la teoría crítica (Brohm, 1994; Sebrelli, 1981), según la cualel deporte aparece como una instancia de distracción que enajena al sujeto de una experiencia directa de sus condiciones materiales de explotación. En segundo lugar, la teoría figuracional (Elias y Dunning, 1992 [1986]) entiende al deporte como una instancia de realización del proceso civilizatorio en tanto regulación creciente de los impulsos violentos, lo que configuraun territorio mimético donde hay lugar para una experiencia agonística, con altos umbrales de represión de la violencia. En tercer lugar, la teoría de la práctica (Bourdieu, 1990; 2000) estabiliza analíticamente al fenómeno deportivo mostrando que se organiza con la lógica de un campo, donde intervienen actores encaramados en luchas incesantes por la apropiación de recursos (capitales) que consideran valiosos al encontrarse encantados por una illusio, propia del campo y anterior a toda posibilidad de elección.Estosactores, a su vez, están gobernados por unos esquemas (habitus) que los ajustan eficientemente a lo que solicitan las situaciones estructurales que los atraviesan sin descanso. Finalmente, las teorías meritocráticas (Ehrenberg, 1991; Bromberger, 1995), que conceptualizan al deporte desde el mito igualitarista por analogía con el imaginario representado como igualitario por los regímenes democráticos, según el cuallos sujetos arribarían al mundo (y al deporte) en igualdad de condiciones, regulados por códigos normativos que garantizarían dicha igualdad, y que admitirían el establecimiento de jerarquías y ascensos sociales (y deportivos) solamente sobre la base del mérito de los participantes.

Por otra parte, las producciones de circulación masiva sobre el deporte, y en particular sobre el fútbol, han declinado con frecuencia hacia dos posiciones normativas: las exegéticas y las denigratorias (Alabarces, 2011). En espejo con el proceso de creciente deportivización de las sociedades contemporáneas emergieron discursos que,al manifestarseen modulaciones periodísticas, políticas, literarias y artísticas (y que muchas veces resonaron en las producciones académicas) hicieron del fenómeno deportivo un objeto de culto, 
acudiendo, y al mismo tiempo dando forma, a las expectativas de los diferentes públicos consumidores. En dirección contraria, pero solidaria con la anterior, en tanto fenómeno popular y de masas el deporte ha sido denunciado como instancia que captura las conciencias de practicantes y consumidores en ensoñaciones de pasatismo y distracción que vehiculizan las energías vitales de los sujetos, quienes de otro modo hubieran tenido un destino de crítica y transformación social. Ocasionalmente, en tanto conciencia crítica de la modernidad y alerta frente a todas las formas de dominación y asimetría existentes, la disciplina sociológica ha sido sensible a este último sentido, activando juicios antes que investigaciones, y ubicando al deporte en el terreno del nuevo opio de los pueblos, (pos)religioso.

$\mathrm{Si}$ es cierto que dentro del repertorio de las especialidades en sociología, el fenómeno deportivo y su estudio son investidos recurrente y disimuladamente de significaciones inferiorizantes, también es cierto que este estigma simbólico aparece contestado en el plano institucional y material a partir de la estabilización de proyectos y carreras de investigación, becas, mesas en congresos y jornadas, publicaciones, espacios curriculares en grado y posgrado abocados al tema, como oportunamente señalaran Alabarces (2011) y Rodríguez (2013), y como el propio Alabarces nos remarca en la entrevista incluida en este número de Cuestiones de Sociología.Si tomamos esto en cuenta, el presente dosier puede leerse como una noticia del estado de asentamiento y estabilización del campo de los estudios sociales del deporte y de su creciente especialización. Y, al mismo tiempo, puede leerse como un mapa de producciones que, al tomarse en serio a los mundos deportivos como objeto de la investigación social, se presenta como un conjunto de operaciones heurísticas, comparativas y teóricas capaces de producir conocimiento empírico sobre las singularidades deportivas, y de producir conocimiento sociológico a partir y desde (no contra) ellas, en diálogo/tensión con los acuerdos teóricos y paradigmáticos de cada época.

En este estado de la discusión, entonces, el dosier compila una serie de trabajos que, desde distintos enfoques disciplinarios como la sociología, la antropología y la historia, se sumergen tanto en las especificidades de las prácticas deportivas, como en los intersticios de la polisémica categoría deporte. La fuerte presencia de trabajos sobre fútbol en esta compilación (¿podría ser de otra manera en una sociedad como la nuestra, y con la proximidad del comienzo del mundial de fútbol a desarrollarse en Rusia?) se enmarca al mismo tiempo en la lógica propia del desarrollo de los estudios sociales del deporte en Latinoamérica, que tuvieron como centro de atención desde sus comienzos al fenómeno futbolístico (Alabarces 2005; Garriga Zucal 2015; Curi,Garriga y Levoratti 2018), y dieronpaso más recientemente a la exploración de otras prácticas deportivas como el rugby, la natación, el golf, el fitness, entre otros. El presente dosier asume la necesidad de retornar al estudio de los mundos del fútbol a partir de una serie de operaciones (comparativas, historiográficas e interpretativas) que permitan ampliar y profundizar el estado del conocimiento sobre dicha práctica, sobre el deporte en general y sobre las configuraciones sociales que el deporte contribuye a entramar y expresar.

Por lo antes dicho, entonces, el proceso de creciente mercantilización del fenómeno futbolístico es abordado por el antropólogo francés Christian Bromberger, quien pone el foco en el modo en que las asimetrías presupuestarias entre los equipos redundan en asimetrías deportivas, cuyo efecto es la desdramatización de la experiencia futbolística por el camino de la neutralización de la incertidumbre y el azar en el resultado. A su vez, Bromberger da cuenta del proceso de deslocalización y la espectacularización de las aficiones a escala europeaalretomarel estudio en torno a una de las dimensiones clásicas de los estudios sociales del deporte relacionada con la producción y transformación de las aficiones deportivas. En esta dirección profundiza el trabajo en colaboración de Buarque, Cabrera, Magazine y Rodríguez quienes desarrollan un estudio comparativo de escala regional sobre las transformaciones recientes de los aficionados organizados en Argentina, Brasil, Costa Rica y México. La metodología comparativa les permite poner de relieve las constantes y las particularidades nacionales en torno a los procesos de mercantilización del fútbol, de estigmatización de las aficiones, de producción de moralidades en torno a las formas violentas del aliento a los clubes, la configuración de identidades y las políticas de pacificación y mediatización de las aficiones, a la 
conformación de estructuras organizacionales en el interior de las hinchadas y la emergencia y sustitución de liderazgos, entre otras dimensiones clave.

La representación del deporte como una expresión de las transformaciones que habrían conducido a la configuración social moderna ha sido un tópico recurrente en la literatura especializada. Frydenberg y Sazbón problematizan aquí, a partir del caso argentino, la linealidad de la identificación entre deporte y modernidad. Por su parte, la asociación entre deporte e integración social ha sido recurrentemente tematizada desde las políticas públicas, los medios masivos de comunicación y, en algunos casos, desde la investigación social. El dosier propone explorar el fenómeno en diferentes dimensiones. El trabajo de la antropóloga brasileña Simoni Guedes, fundadora del campo de estudios sociales del deporte en Latinoamérica, estudia la lógica de emergencia de proyectos de intervención social a través del deporte desarrollados por deportistas o exdeportistas mundialmente célebres, ofreciendo una clave interpretativa del fenómeno a partir de la teoría maussiana del don. Por su parte, Federico Czesli se interroga sobre el modo en que el deporte es investido de sentidos morales en una "comunidad indígena" de Argentina,en la quese implementan políticas públicas de prevención y control de adicciones a través del deporte. Por último, con el foco puesto en las políticas públicas deportivas, Verónica Moreira y Alejo Levoratti dan cuenta de las transformaciones en los marcos normativos y de su implementación, que tuvieron lugar en Argentina a partir del cambio de gobierno en 2015.

La nota de investigación en colaboración de Garriga, Hang y Iuliano pone en diálogo los hallazgos de tres investigaciones en torno a los practicantes de golf, de natación y los hinchas de fútbol, con el objeto de pensar el modo en que el deporte aparece unas veces como instancia que permite regular y gestionar emociones, y otras veces como espacio que habilita la emergencia de las mismas, las cuales adquieren racionalidades específicas que sólo se comprenden a partir de las lógicas propias de las prácticas deportivas de las que se trate. De este modo, la puesta en relación de hallazgos empíricos en los distintos escenarios deportivos habilita un pasaje hacia la pregunta teórica sobre las emociones en las sociedades contemporáneas.

En este dosier Diego Roldán encuentra en el mundial 78 un acontecimiento extraordinario tanto en la historia represiva de la dictadura como en el devenir de la espectacularización del fútbol, en el cual la tensión entre la emergencia y regulación de las pasiones de los hinchas cobra una forma específica bajo el gobierno dictatorial. En efecto, el mundial 78 aparece para Roldán como una "pieza de abordaje incómodo", que propone un enlace complejo entre política, autoritarismo, nacionalismo, deporte y culturas populares. A partir del análisis de sus condiciones de posibilidad, de las convergencias de las tecnologías de gobierno militar y de gobierno deportivo, y del desarrollo de movimientos y corporalidades en la ceremonia inaugural y festejos callejeros, el texto ilumina-enraizado en el fútbol- los vínculos entre las macropolíticas económicas y sociales, y el gobierno de la selección.

Si bien la dimensión de género no ha estado ausente en las producciones que estructuraron el campo en los estudios sociales del deporte, su conceptualización y su elaboración empírica en torno a diferentes prácticas e instituciones deportivas se encuentra en desarrollo. Los trabajos de Alvarez Litke e Hijós representan un aporte en esa dirección, a la vez que elaboran y sistematizan un mapa de lectura sobre las investigaciones recientes sobre género y deporte (Hijós), y sobre fútbol femenino (Álvarez Litke).

La entrevista a Pablo Alabarces nos invita a recorrer la constitución del campo de los estudios sociales del deporte desde su propia biografía. Tal historización propone un camino que comienza en la periferia de nuestra academia, pero que tiene desarrollos producto de los hallazgos del trabajo empírico que han llevado al campo a una relativa consolidación. Resta una mejor articulación con las políticas públicas deportivas, y una profundización de investigaciones empíricas que descentren a la disciplina de los mundos del fútbol. En ese camino, la sección "lecturas" del dosier presenta una ventana a la producción reciente sobre los estudios sociales del deporte, en el plano local y latinoamericano. Kopelovich reseña el libro compilado por Levoratti y Moreira Deporte, Cultura y Sociedad. Estudios socio-antropológicos en Argentina, mientras que Rosa y Rueda presentan la compilación a cargo de los investigadores chilenos Soto Lagos y Fernández Vergara ¿Quién raya la cancha? Visiones, tensiones y nuevas perspectivas en los estudios socioculturales del deporte en 
Latinoamérica. Si bien en ambas producciones, como señalan Rosa y Rueda, el fútbol es el deporte dominante, la emergencia de investigaciones en torno a prácticas, tales como el rugby, la natación o el ciclismo, queda plasmada en las compilaciones.

Un tema que aparece con recurrencia en las agendas de investigación en torno al deporte se relaciona con la transferencia y vinculación entre los conocimientos fruto de la investigación, la intervención y el diseño de políticas públicas. Los trabajos aquí reunidos permiten formular interrogantes sobre los programas relacionados con el "deporte social” y su carácter inclusivo ¿Qué sentidos condensa la categoría social? ¿Existe alguna manifestación deportiva que no sea al mismo tiempo "social", incluso en el caso de los "deportes de elite" (categoría que en este contexto asume un sentido profesionalizante, más que clasista) respecto de los cuales esta categoría demarca su frontera? Las contribuciones del presente dosier habilitan una interrogación sobre la asimilación entre el deporte concebido como "social" (o "comunitario") y las políticas deportivas proyectadas sobre las poblaciones pobres. Los objetivos de "inclusión" que habitan a los diseños del deporte "social" activan a su vez ambiciones regulatorias (al monopolizar la definición legítima de deporte, actividad física, salud, frente a los juegos y pasatiempos locales), e incluso civilizatorias, en los términos de Elias (al promover entre los "excluidos" formas de autoregulación de la violencia), y moralizantes (al habilitar valoraciones sobre lo que es apropiado y conveniente que determinadas poblaciones hagan con su tiempo libre). Estos interrogantes se sitúan, a la vez, en adyacencia a la inquietud por las posibilidades de mejorar las condiciones de vida y reducir las desigualdades sociales y ciudadanas a través de las políticas públicas.

Por último, los trabajos aquí reunidos representan una clave de acceso posible para la comprensión sociológica de las formas en que se configuran contemporáneamente las categorías del apasionamiento, las emociones, las aficiones, el sacrificio, el uso del "tiempo libre”, la filantropía y las políticas de inserción, las representaciones sobre la salud y lo saludable, los imaginarios de clase y género, los entramados políticos constitutivos de las formas estatales en su sentido amplio (es decir, empírico), que son invisibilizados con frecuencia desde las teorías (normativas) de la política, entre otros. Además, el dosier ilumina, en un año en el quemuchas miradas se enfocan en el mundial, varios de los interrogantes que las ciencias sociales han estudiado en el deporte. Las operaciones que hacen de una competencia deportiva de varones un evento que refiere a la nación, a los estereotipos de género y que se presenta en muchos ocasiones como una cuestión de Estado desnudan un objeto sociológico pujante e inabarcable. En este dosier, abordado desde la sociología, la antropología y la historia, el deporte se transforma en un objeto valioso para reflexionar, también, sobre las apuestas metodológicas variadas y variables. Reflexión que permite dar cuenta de la necesidad de articular diferentes enfoques para enriquecer saberes y perspectivas. Si podemos dejar atrás la mirada reduccionista que no ve en el deporte otra cosa que no sea, o bien una constatación de lo que tenemos resuelto teóricamente de antemano, o bien un territorio de manifestación de luchas sociales que tienen su epicentro en otra localización social y que repercuten en el campo deportivo sólo para simbolizarse, posiblemente consigamos contribuir a la configuración de nuevas formas y nuevos rumbos para la imaginación sociológica.

\section{REFERENCIAS}

Alabarces, P. (2005). Hinchadas. Buenos Aires: Prometeo

Alabarces, P. (2011). Veinte años de ciencias sociales y deportes, diez años después”. Revista de ALESDE. Curitiba, UFPR, año $1 \mathrm{n}^{\circ} 1,11-22$.

Bourdieu, P. (1990). ¿Cómo se puede ser deportista?”. En Sociología y Cultura (pp.193-213). Méjico: Grijalbo.

Bourdieu, P. (2000). Programa para una sociología del deporte. En Cosas dichas (pp. 173-185). Barcelona: Gedisa

Brohm, J.M. (1994). 20 tesis sobre el deporte. En AAVV, Materiales de sociología del deporte (pp. 47-55). Madrid: Ediciones de la Piqueta.

Bromberger, Ch. y Hayot, A. (1995). Le Match de football: Ethnologied'une passion partisane à Marseilles, Naples et Turin. Paris: Éditions de la MSH. 
Curi, M., Garriga Zucal, J y Levoratti, A. (2018). Deporte(s) y Antropología: enfoques, objetos y prácticas. Repensando sus configuraciones en Suramérica. Antípoda. Revista de Antropología y Arqueología. 30: 1-16. doi: https:// dx.doi.org/10.7440/antipoda30.2018.01

Elias, N. y Dunning, E. (1992) [1986]. Deporte y ocio en el proceso civilizatorio. México: FCE.

Ehrenberg, A (1991). Le culte de la performance. Paris: Calmann-Lévy.

Garriga Zucal, J. (2015).Violencia en el fútbol: investigaciones sociales y fracasospoliticos. Buenos Aires: Ediciones Godot.

Rodriguez, M. G. (2013), ¿Qué es un campo, y tú me lo preguntas?. En Branz, Juan, Garriga Zucal, José y Moreira, Verónica (comp) Deporte y ciencias sociales. Claves para pensar las sociedades contemporáneas. La Plata: EDULP.

Sebrelli, J. J. (1981). Fútboly masas. Buenos Aires: Galerna. 